\title{
Chronic Unpredictable Stress Induces a Cognitive Deficit and Anxiety-Like Behavior in Rats that is Prevented by Chronic Antidepressant Drug Treatment
}

\author{
Corina O Bondi', Gustavo Rodriguez', Georgianna G Gould', Alan Frazer' and David A Morilak*,' \\ 'Department of Pharmacology and Center for Biomedical Neuroscience, University of Texas Health Science Center, San Antonio, TX, USA
}

\begin{abstract}
Chronic stress is a risk factor for the development of many psychopathological conditions in humans, including major depression and anxiety disorders. There is a high degree of comorbidity of depression and anxiety. Moreover, cognitive impairments associated with frontal lobe dysfunction, including deficits in cognitive set-shifting and behavioral flexibility, are increasingly recognized as major components of depression, anxiety disorders, and other stress-related psychiatric illnesses. To begin to understand the neurobiological mechanisms underlying the cognitive and emotional consequences of chronic stress, it is necessary to employ an animal model that exhibits similar effects. In the present study, a rat model of chronic unpredictable stress (CUS) consistently induced a cognitive impairment in extradimensional set shifting capability in an attentional set shifting test, suggesting an alteration in function of the medial prefrontal cortex. CUS also increased anxiety-like behavior on the elevated plus-maze. Further, chronic treatment both with the selective norepinephrine reuptake blocker, desipramine $(7.5 \mathrm{mg} / \mathrm{kg} /$ day), and the selective serotonin reuptake blocker, escitalopram (I0 mg/kg/ day), beginning I week before CUS treatment and continuing through the behavioral testing period, prevented the CUS-induced deficit in extradimensional set-shifting. Chronic desipramine treatment also prevented the CUS-induced increase in anxiety-like behavioral reactivity on the plus-maze, but escitalopram was less effective on this measure. Thus, CUS induced both cognitive and emotional disturbances that are similar to components of major depression and anxiety disorders. These effects were prevented by chronic treatment with antidepressant drugs, consistent also with clinical evidence that relapse of depressive episodes can be prevented by antidepressant drug treatment.
\end{abstract}

Neuropsychopharmacology (2008) 33, 320-331; doi:I0.1038/sj.npp. I30I4I0; published online 4 April 2007

Keywords: antidepressants; anxiety; chronic stress; cognition; desipramine; escitalopram

\section{INTRODUCTION}

Chronic exposure to stressful life events is an established risk factor for the development of many psychological conditions in humans, including major depression (Kendler et al, 1998, 1999). Further, it is becoming increasingly clear that cognitive and emotional biases play an important role in the development and maintenance of depression, especially in response to stress (Anisman and Matheson, 2005; Beck, 1976; Coles and Heimberg, 2002; Mathews and Mackintosh, 1998). Based on clinical neuropsychological studies, cognitive impairments associated with frontal lobe executive function (ie cognitive set-shifting, behavioral flexibility, and perseveration) have been identified in depression and anxiety disorders (Anisman and Matheson,

*Correspondence: Dr DA Morilak, Department of Pharmacology, MC 7764, University of Texas Health Science Center at San Antonio, 7703 Floyd Curl Drive, San Antonio, TX 78229-3900, USA, Tel: + I 210567 4174, Fax: + I 210567 4303, E-mail: morilak@uthscsa.edu Received 3 January 2007; revised 2 March 2007; accepted 5 March 2007
2005; Fossati et al, 1999; Murphy et al, 1999). Depressed patients show altered responses on verbal fluency and attentional set-shifting tasks (Austin et al, 2001; Beats et al, 1996; Purcell et al, 1997), as well as diminished motivation and impaired extradimensional set shifting (Miller and Lewis, 1977; Murphy et al, 1999). Consistent with such neuropsychological findings, imaging studies have shown hypoactive function of prefrontal cortex in depression (Rogers et al, 2004; Sheline, 2003), which was improved upon treatment with antidepressant drugs, such as paroxetine (Kennedy et al, 2001; Prasko et al, 2004).

There is a high degree of comorbidity of depression and anxiety disorders, with symptomatology reflecting affective, somatic and cognitive dysregulation associated with both depression and anxiety (Mineka et al, 1998; Morilak and Frazer, 2004). However, although many pre-clinical studies attempting to model aspects of depression have focused on behaviors thought to represent anhedonia, reduced locomotor activity or behavioral despair (eg, Lu et al, 2006; Rygula et al, 2005; Willner, 1997; Willner and Mitchell, 2002), relatively few have attempted to model cognitive impairments or state anxiety associated with depression. 
Unpredictable stressors have greater negative impact in humans than predictable ones, perhaps due to temporal uncertainty and inability to anticipate the event (see Anisman and Matheson, 2005; Willner and Mitchell, 2002). Thus, in the first experiment of the present study, both cognitive dysregulation and anxiety-like behavioral alterations induced by the chronic unpredictable stress (CUS) model of depression were assessed. We determined whether 2 weeks of CUS would induce cognitive deficits in the performance of adult male Sprague-Dawley rats on an attentional set shifting test (AST), or increase anxiety-like behavioral reactivity tested on the elevated-plus maze (EPM).

Attentional set-shifting reflects an ability to 'unlearn' an established contingency in order to learn a new one by shifting attention from a salient stimulus dimension to one that was previously irrelevant. This has been shown to be mediated by the medial prefrontal cortex (mPFC) in rats (Birrell and Brown, 2000). In humans, the Wisconsin Card Sorting Test (WCST) has been used to assess strategyswitching deficits in patients with frontal lobe damage, depression, and other neuropsychiatric conditions thought to involve the prefrontal cortex (Merriam et al, 1999; Stuss et al, 2000; Tollefson, 1996). To address more specifically the different components of behavioral flexibility assessed in the WCST, Roberts et al (1992) developed a visual discrimination task to investigate attentional set formation in both humans and primates. Birrell and Brown (2000) subsequently developed a similar test to assess attentional set shifting performance in rats. In this test, rats are trained to dig for a food reward in small pots containing distinctive digging media and marked with different odors. The rats must learn which of these stimulus dimensions (odor or digging medium) is salient in signaling the food reward. After learning a given contingency, the rules are changed as in the WCST, and the rats must unlearn the previous contingency to learn a new one. In this way, the rats are tested on a series of increasingly difficult tasks including stimulus reversals, and intra- and extra-dimensional (ED) set shifts. Using this test, lesions of rat mPFC were shown to interfere specifically with ED set shifting capability, reflecting an inability to attend to a previously irrelevant stimulus dimension and ignore the previously salient dimension that is no longer informative (Birrell and Brown, 2000).

The second experiment then tested whether any cognitive and/or anxiety-like behavioral alterations induced by CUS were sensitive to chronic treatment with antidepressant drugs of two different classes, a selective NE reuptake inhibitor (NRI), desipramine (DMI), or a selective serotonin reuptake inhibitor, escitalopram (SCIT). Portions of this work have been presented in abstract form (Bondi et al, 2006).

\section{MATERIALS AND METHODS}

\section{Animals}

A total of 85 adult male Sprague-Dawley rats (Harlan, Indianapolis, IN, USA), weighing 200-250 g upon arrival, were initially housed in groups of three in $25 \times 45 \times 15 \mathrm{~cm}$ plastic cages, and maintained on a 12/12 h light/dark cycle (lights on at $0700 \mathrm{~h}$ ), with food and water available ad libitum. They were allowed to acclimate to the housing facility for at least 4 days before use in any experimental or surgical procedures. In addition, for the social defeat procedure, two male Long-Evans rats (Harlan, Indianapolis, IN, USA), that weighed $400-450 \mathrm{~g}$ were used as resident attackers. These rats were each paired with an ovariectomized female and housed in a separate room in large cages $(80 \times 55 \times 40 \mathrm{~cm})$, on the same light-dark cycle. All experiments were carried out during the light portion of the cycle, between 0800 and $1700 \mathrm{~h}$. All procedures were conducted according to NIH guidelines for the care and use of laboratory animals, and were reviewed and approved by the Institutional Animal Care and Use Committee of the University of Texas Health Science Center at San Antonio. All efforts were made to minimize animal pain, suffering or discomfort, and to minimize the number of rats used.

\section{Experiment 1: Effect of Chronic Unpredictable Stress on Attentional Set-Shifting and Anxiety-Like Behavior on the Elevated Plus-Maze}

A group of 34 rats were randomly assigned to either the CUS group or unstressed control group ( $n=17$ /group) to assess the effects of chronic stress on attentional set-shifting performance and on state anxiety in the elevated plus-maze test. Animals were weighed every 3 days during the stress and testing procedures. In this experiment and in experiment 2 , investigators conducting the behavioral tests were blind to the treatment conditions of the animals.

\section{Chronic Unpredictable Stress Procedure}

The CUS procedure is a modification of published procedures (Katz et al, 1981; Lu et al, 2006; Willner et al, 1987). One day before beginning the chronic unpredictable stress, rats were housed individually, and the CUS procedure was then applied for 14 consecutive days, as outlined in Table 1. This paradigm was devised to maximize unpredictability, in that the stressors were applied in seemingly random order and at varying times during the light phase (0800-1700 h). All procedures were carried out in isolated rooms adjacent to the housing room, requiring minimal handling or transport of the rats. After each stressor, animals were kept in a recovery room for $1-2 \mathrm{~h}$, following which they were placed in clean cages with fresh bedding and returned to the housing facility. Control rats were individually housed for the same period of time, and were handled daily for $30 \mathrm{~s}$ in the housing room, but were not stressed.

Specific details of the CUS procedure are as follows: for 30-min restraint, rats were placed in a restraining device made of Plexiglas and flexible nylon, which was closed using two Velcro strips, thus restricting movement but allowing free respiration and air circulation. The shaking-crowding procedure was carried out by placing six rats in a cardboard box atop a lab shaker set to produce 220 back-and-forth movements (approximately 2 -inch sideways deflection) per min. The resident-intruder social defeat was performed as described previously (Nikulina et al, 2005; Ruis et al, 1999). The 'intruder' Sprague-Dawley test rat was transported to the Long-Evans residents' housing room. The ovariectomized female was removed from the resident cage 
Table I Chronic Unpredictable Stress Experimental Schedule

\begin{tabular}{|c|c|}
\hline Day 1 & 30-min restraint \\
\hline Day 2 & I-h shaking/crowding \\
\hline Day 3 & Social defeat \\
\hline Day 4 & I5-min warm water swim \\
\hline Day 5 & 24-h high density housing \\
\hline Day 6 & 10-min cold water swim \\
\hline Day 7 & I-h shaking/crowding \\
\hline Day 8 & I5-min mild footshock \\
\hline Day 9 & Social defeat \\
\hline \multirow[t]{2}{*}{ Day 10} & I5-min warm water swim \\
\hline & (begin food restriction for AST) \\
\hline Day II & I5-min mild footshock \\
\hline Day 12 & 10-min tail pinch in restrainer \\
\hline Day 13 & 10-min cold water swim \\
\hline Day 14 & I5-min mild footshock \\
\hline Day 15 & Habituation day for AST \\
\hline Day 16 & Training day for AST \\
\hline Day 17 & Testing day for AST \\
\hline Day 18 & Testing day for elevated plus-ma \\
\hline
\end{tabular}

Experimental schedule for the chronic unpredictable stress procedure, including the timing for the attentional set shifting and elevated plus maze tests. For rats subjected to chronic antidepressant drug treatment (experiment 2), osmotic minipumps were implanted 8 days before beginning the CUS procedure.

immediately before introducing the intruder. Within approximately $2-5 \mathrm{~min}$, the intruder was attacked and defeated, indicated by fleeing, freezing, and submissive behavior. When full submissive posture occurred, as indicated by the intruder lying motionless on its back for $4 \mathrm{~s}$, it was separated from the resident, protected from further attacks by placing it within a small wire mesh cage $(30 \times 15 \times 15 \mathrm{~cm})$, and kept in the resident cage for the remainder of the $45 \mathrm{~min}$ session. This allowed for continuing auditory, olfactory, and visual contact between the two rats. The defeated rat was then removed and returned to its home cage and the Long-Evans female returned to the resident cage. Warm swim and cold swim were accomplished by placing the rat in a cylindrical tank $(60 \mathrm{~cm}$ height $\times 30 \mathrm{~cm}$ diameter) filled with water to a $30 \mathrm{~cm}$ depth at 25 or $18^{\circ} \mathrm{C}$, respectively. High-density housing was 6 rats per cage for $24 \mathrm{~h}$. Electric footshock consisted of $1.5 \mathrm{~mA}$ scrambled shock delivered through the grid floor of a chamber enclosed within a skinner box (30 s on, $150 \mathrm{~s}$ off, for $15 \mathrm{~min}$ ) except on the last day of the CUS procedure, during which the shock was applied $5 \mathrm{~s}$ on, $175 \mathrm{~s}$ off, for 15 min (Table 1). Finally, tail pinch involved placing the rat in the previously described restraining device, and applying a clothespin $1 \mathrm{~cm}$ from the base of the tail for $10 \mathrm{~min}$.

\section{Attentional Set-Shifting Protocol}

Procedures for the attentional set-shifting test were as described previously (Lapiz and Morilak, 2006), adapted from Birrell and Brown (2000). On day 10 of the CUS procedure, ie, 1 week before testing, both CUS rats and their unstressed control counterparts were placed on a restricted diet of $14-15 \mathrm{~g}$ of food (four pellets) per day, qwith water still freely available. The testing apparatus was a custombuilt white rectangular wooden arena $(45 \times 71 \times 22 \mathrm{~cm})$, painted white on all surfaces. A removable white divider separated one-third of the length of the arena from the rest, forming a start box, which also served as a holding area following each trial, allowing the experimenter to clean the arena and change pots. To begin each trial, a rat was placed in the start box, and given access to the rest of the arena by lifting the divider. A white Plexiglas panel divided the opposite third of the arena into two sections. At testing, one digging bowl was placed in each section. The separation enabled the experimenter to quickly remove the rat following a response, preventing it from moving to the other bowl. The digging bowls consisted of small terracotta pots (internal rim diameter $7 \mathrm{~cm}$; depth $6 \mathrm{~cm}$ ). Each pot was defined by a pair of cues along two stimulus dimensions, namely the digging medium with which the pot was filled and an odor (see Table 2). To mark each pot with a distinct odor, $20 \mu \mathrm{l}$ of scented aromatic oil (Frontier Natural Brands, Boulder, CO, USA) were applied initially to the inner rim, and then $2 \mu \mathrm{l}$ were reapplied twice a week to maintain a consistent odor. A different pot was used for each combination of digging medium and odor, and only one odor was ever applied to a given pot. The bait, which was placed in the bottom of the 'positive' pot and buried with the digging medium, was a $1 / 4$ Honey Nut Cheerio (General Mills Cereals, Minneapolis, MN, USA). In all discrimination trials, a small quantity of powdered Cheerio was sprinkled onto the digging media to eliminate the possibility that the rat may locate the reward by smell rather than by learning the discrimination.

Digging was defined as a vigorous displacement of the medium to retrieve the reward buried within the pot. Simply investigating the rim of the pot or the surface of the digging medium with paws or snout without displacing material was not scored as a 'dig', and the trial continued until a 'dig' response was executed. Thus, rats were able to access tactile, visual and olfactory characteristics of the pots to make their choices based on these stimulus dimensions. Food restriction was continued for 7 days through the day of testing. Beginning after 4 days of food restriction, the behavioral procedure was conducted over three days for each rat as follows:

\section{Day 1: Habituation Day}

The rats were first trained to dig reliably for food reward in the pots. Two unscented pots were placed in their home cage and rebaited every $5 \mathrm{~min}$, with the cheerio covered with increasing amounts of sawdust (three times with no sawdust, three times with the pots one-third full of sawdust, three times with the pots $\frac{1}{2}$ full and three times completely full). Once the rat successfully retrieved the cheerio from the pots each time they were baited, it was transferred to the testing arena and given three consecutive trials to retrieve the reward from both sawdust-filled pots.

\section{Day 2: Training Day}

On the next day, animals were trained to complete a series of simple discriminations (SD), to a criterion of six 
Table 2 Behavioral Protocol for Attentional Set Shift Testing

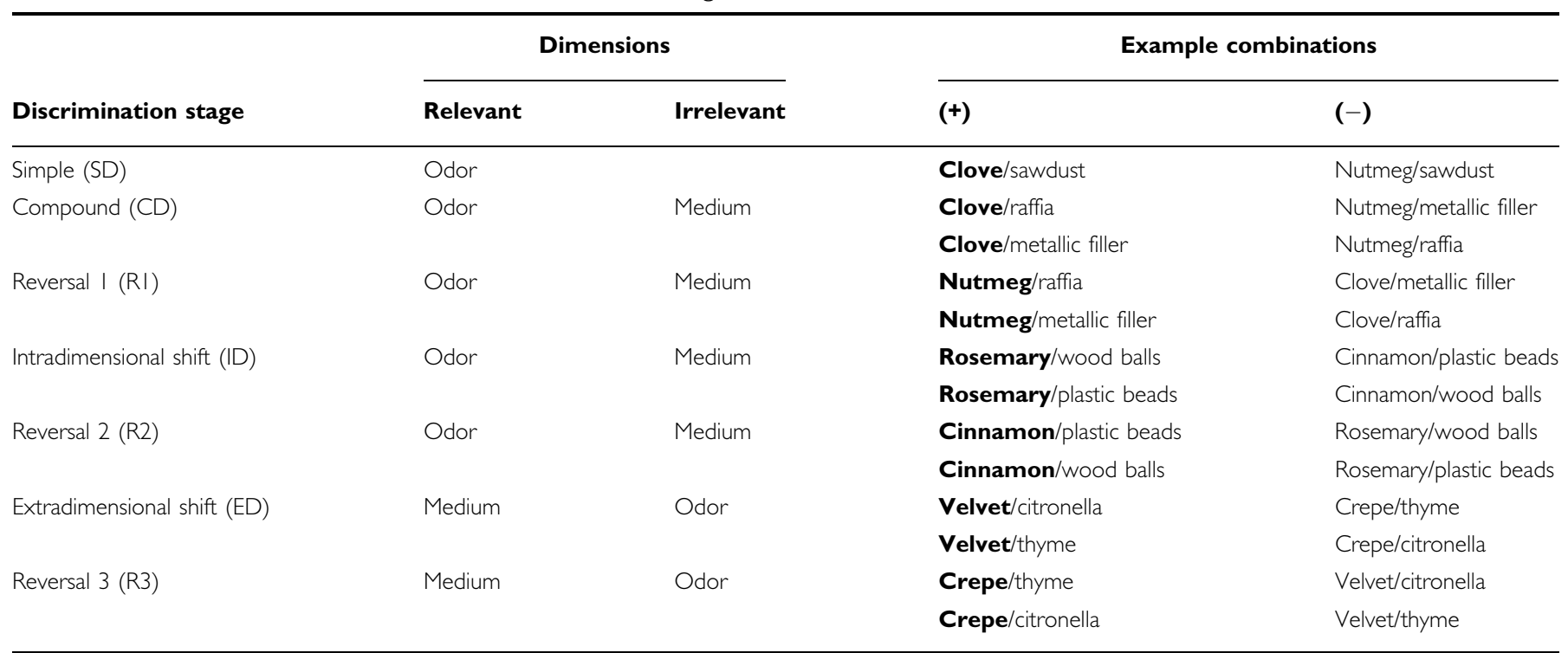

Representative example of stimulus pairs and the progression through the stages of the attentional set shifting protocol. In this example, odor was the initial

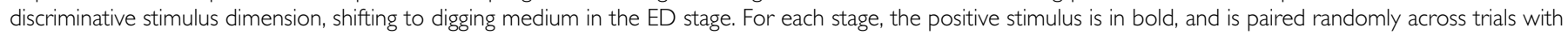
the two stimuli from the irrelevant dimension.

consecutive correct trials each. First, both pots were filled with the same medium (sawdust), and the bait paired with one of two odors (lemon $v s$ rosewood). After reaching criterion, new unscented-pots were introduced, and the rat had to learn to associate the food reward with one of the digging media in the bowls (felt strips $v s$ shredded paper). All rats were trained using the same stimulus exemplars, and in the same order. The positive and negative cues for each rat were randomly determined and equally represented. The training exemplars were not used again during testing.

\section{Day 3: Testing Day}

Following the training day, the animals were tested on a series of increasingly difficult discriminations (Table 2). To proceed to the next stage, the rat had to reach a criterion of six consecutive correct trials. At each stage of testing, the discriminative stimulus dimension and the positive cue within that dimension were varied according to the contingency schedule shown in Table 2. The first task was a simple discrimination (SD), similar to the training trials, involving only one stimulus dimension. Half the rats were required to discriminate between two odors, only one of which was associated with reward, with both pots filled with sawdust. For the other half, this first discrimination involved digging media, and the pots were unscented (for clarity, the remainder of this description will only consider the example beginning with odor discrimination). The second stage was then a compound discrimination (CD), in which the same discrimination was required (eg, odor), but the second, irrelevant stimulus was introduced. As in the SD task, only one odor was associated with reward and two different digging media were paired randomly with the odors. The third stage was a reversal of the previous discrimination (R1), in which the same odors and media were used, and odor was still the relevant dimension, but in these trials the negative odor from the previous stage was now positive (ie, associated with the reward), and the positive odor from the previous stage was now negative (no reward). The fourth stage was the first dimensional shift, in which all new stimuli (odors and media) were introduced. This task was an intradimensional (ID) shift because odor was still the relevant dimension and medium still irrelevant. The fifth stage was a reversal of this discrimination (R2), in which the previously negative odor was now positive, similar to R1. The sixth stage required an extradimensional (ED) shift, in which all new stimuli were again introduced, but this time the relevant dimension was also changed, for example, the digging medium became the relevant dimension and odor was now irrelevant. Finally, the seventh stage was another reversal (R3), in which the previously negative medium was now positive. The assignment of each exemplar in a pair as being positive or negative in a given stage, as well as the left-right positioning of the pots in the arena on each trial, were determined randomly in advance. As described above, half the rats were first presented with odor as the relevant cue, and for half the discrimination began with medium as the salient cue. Table 2 outlines the progression through these stages, and provides examples of the cue combinations used, beginning in this case with an odor discrimination, shifting to medium in the ED shift stage. The dependent measure in this procedure was the number of trials required to reach the criterion of six consecutive correct responses at each test stage (Trials to Criterion, TTC).

\section{The Elevated Plus-Maze Test}

The elevated plus-maze test was carried out as described previously (Cecchi et al, 2002a, b; Pardon et al, 2002), on the day following the AST (see Table 1). Rats were transported to the testing room in their home cage, and allowed to acclimate for $15 \mathrm{~min}$. Continuous background white noise $(60 \mathrm{~dB})$ was presented through stereo speakers in the room. The elevated plus-maze (Accuscan Instruments, Columbus, 
$\mathrm{OH})$ consists of four white plastic arms, $10 \times 50 \mathrm{~cm}$, oriented in the shape of a cross, intersecting at a $10 \times 10 \mathrm{~cm}$ platform in the middle. Two arms situated opposite of each other are enclosed by walls $48 \mathrm{~cm}$ high ('closed arms'). The remaining two 'open arms' have no walls, but are fitted with a $0.5 \mathrm{~cm}$ clear plastic rim around the edges to prevent animals from falling off. The maze is elevated $100 \mathrm{~cm}$ from the floor, and testing took place under normal ambient overhead lighting (200 lux measured in the open arms). The rat was placed in the center platform, facing a junction between a closed and an open arm, and allowed to explore freely for $5 \mathrm{~min}$. Dual infrared sensor beams positioned at the entry of each arm provided automated measures of time spent and number of entries made in each of the arms, as well as the central platform. Open to total ratios (OTR) for time and entries were calculated as indices of open-arm exploration, defined as the proportion of time spent or entries made in the open arms relative to the total time or entries made into all arms (open/open + closed). Total arm entries and closed-arm entries were both analyzed as measures of non-specific changes in locomotor activity independent of OTR.

\section{Experiment 2: Effect of Chronic Antidepressant Drug Treatment in Preventing the CUS-Induced Alterations on Attentional Set-Shifting and Behavioral Reactivity on the Elevated Plus-Maze}

Fifty-one rats were randomly assigned to six groups ( $n=$ 8-9/group) defined by chronic stress exposure (control or CUS), as well as three chronic drug conditions: vehicle, DMI or SCIT. Rats were anesthetized with a cocktail of ketamine $43 \mathrm{mg} / \mathrm{ml}$, acepromazine $1.4 \mathrm{mg} / \mathrm{ml}$, xylazine $8.6 \mathrm{mg} / \mathrm{ml}$, $1.0 \mathrm{ml} / \mathrm{kg}$, i.m., with a $25 \%$ supplement given as needed. Osmotic minipumps (model 2ML4, Alzet Corp., Palo Alto, CA), preloaded with either vehicle (10\% ethanol in saline), DMI (Sigma, St Louis, MO) or SCIT (Forest Laboratories, Jersey City, NJ) at concentrations calculated to deliver $7.5 \mathrm{mg} / \mathrm{kg} /$ day of the free base for DMI and $10 \mathrm{mg} / \mathrm{kg} /$ day for SCIT, were implanted intraperitoneally via a ventral midline incision. Following surgery, rats were treated prophylactically with antibiotic (penicillin G, $300000 \mathrm{IU} / \mathrm{ml}, 1.0 \mathrm{ml} / \mathrm{kg}$, s.c.) and housed singly.

On day 8, the CUS procedure was initiated as described in Experiment 1 (see Table 1). Also as described above, rats were placed on a restricted diet on day 17 of chronic drug treatment, then taken through habituation, training, and testing on the attention set-shifting test (day 24) and the EPM test (day 25). Rats were weighed every 3 days throughout the procedure. At the end of the experiment, rats were killed by rapid decapitation, and trunk blood was collected for analysis of plasma DMI and SCIT levels (assays conducted by Dr Martin Javors, Department of Psychiatry, UTHSCSA).

\section{Data Analysis}

During the attentional set-shifting test, the number of trials to criterion was recorded for each stage. In Experiment 1, AST data were analyzed by two-way ANOVA (stress $x$ stage), with repeated measures over stage. Where significant main effects or interactions were indicated, post hoc planned comparisons were made using the Newman-Keuls test. Results from the EPM were analyzed by Student's $t$-test. In Experiment 2, AST data were first analyzed by three-way ANOVA (stress $\times$ drug $\times$ stage), with repeated measures over stage. Significant main effects were analyzed by post hoc comparisons with the Newman-Keuls test. Following a significant three-way interaction, post hoc two-way ANOVAs (stress $\times$ drug) were conducted for each test stage, after which further post hoc comparisons were made within stage using Fisher's LSD test. Results from the EPM in Experiment 2 were analyzed by two-way ANOVA (stress $\times$ drug). To assess the effectiveness and impact of the CUS procedure, body weight data were analyzed by ANOVA, with repeated measures over time. Significance for all analyses was determined at $p<0.05$. Any subject that failed to complete the training stages was eliminated a priori from subsequent testing on the AST, resulting in the elimination of 12 rats from an initial total of 85 (six each from experiments 1 and 2). However, subjects so eliminated from the AST solely for failure to train or complete all test stages were still tested on the EPM the following day, and their body weight data were also analyzed. Thus, they were included in the overall number of rats reported.

\section{RESULTS}

Experiment 1: Effect of Chronic Unpredictable Stress on Attentional Set-Shifting and Behavioral Reactivity on the Elevated Plus-Maze

There was no significant difference between groups for the number of trials required to reach criterion for the SD task on the training day $\left(t_{26}=0.823, p=0.42\right)$. Figure 1 shows the effects of CUS on the trials to criterion (TTC) for each stage of the AST on the testing day. Two-way analysis of variance (ANOVA) (stress $\times$ task) with repeated measures over task revealed significant main effects of stress $\left(\mathrm{F}_{1,26}=\right.$ 19.897, $p<0.001)$ and task $\left(\mathrm{F}_{6,156}=9.277, p<0.0001\right)$, but no stress $\times$ task interaction $\left(\mathrm{F}_{6,156}=1.027, p=0.41\right)$. For the main effect of task, post hoc comparisons showed that significantly more trials were required to reach criterion during R1, R3, and ED than in the other tasks (Figure 1). This is consistent with the relatively greater difficulty of the reversal and ED set-shifting tasks, as reported previously (Birrell and Brown, 2000; Lapiz and Morilak, 2006). Post hoc analysis of the effect of Stress indicated that CUS induced a deficit in performance on the AST, manifest as a significant increase in trials to criterion specifically on the R1 and R3 reversals, and on the ED set-shifting task (Figure 1).

Figure 2 shows the effect of CUS exposure on anxiety-like behavior on the elevated plus-maze. CUS produced anxietylike reductions in open-arm exploration, indicated by significantly lower open-to-total ratio (OTR) values for both time $(t=3.134, p<0.005$, Figure $2 \mathrm{a})$, and entries $(t=3.150, p<0.005$, Figure $2 \mathrm{~b})$. The CUS-induced reduction in open-arm exploration was not associated with reduced exploratory drive or locomotor activity, as there was no difference between groups in either total arm entries $(t=0.605, p=0.5498$, Figure $2 \mathrm{c})$, or closed-arm entries $(t=-1.3, p=0.203$, Figure $2 \mathrm{~d})$.

CUS significantly decreased the rate of body weight gain over the 18 day procedure $(t=2.04, p<0.001)$. Control rats 
gained $1.47 \pm 0.25 \mathrm{~g} /$ day (mean $\pm \mathrm{SEM}$ ), whereas the body weight of rats exposed to CUS changed very little $(-0.01 \pm 0.3 \mathrm{~g} /$ day $)$.

\section{Experiment 2: Effect of Chronic Antidepressant Drug Treatment in Preventing the CUS-Induced Alterations on Attentional Set-Shifting and Behavioral Reactivity on the Elevated Plus-Maze}

Chronic drug treatment resulted in mean plasma DMI and SCIT levels of $183 \pm 28$ and $34 \pm 4 \mathrm{ng} / \mathrm{ml}$, respectively. These values are within clinically relevant therapeutic ranges (Perry et al, 1994; Nikisch et al, 2004). There were no

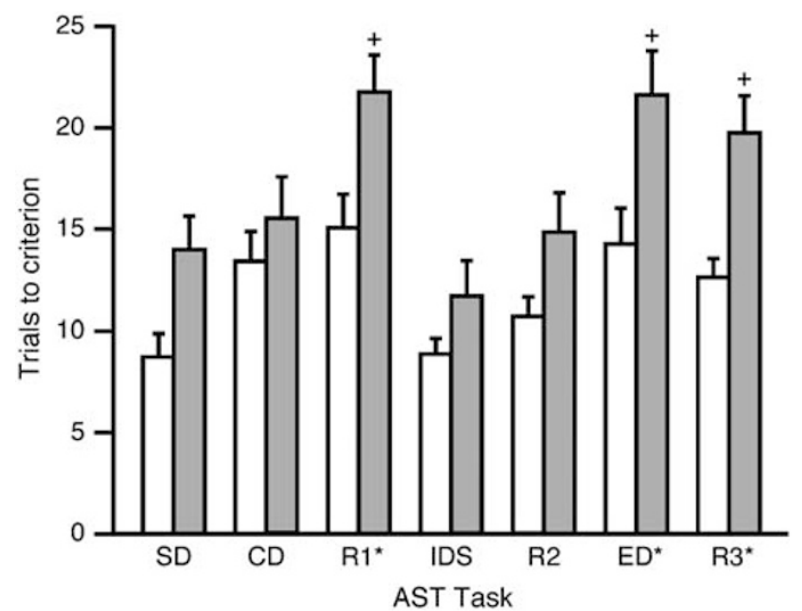

Figure I Effect of CUS on cognitive performance on the attentional set shifting test. Significantly more trials were required to reach criterion on the $\mathrm{RI}, \mathrm{ED}$ and R3 tasks (Main effect of Stage: $* 0<0.05$ compared to the other stages, collapsed across treatment groups; data expressed as mean \pm SEM, $n=14 /$ group). CUS produced a significant deficit in performance on the AST, manifest as a significantly higher number of trials to criterion on RI, $\mathrm{ED}$ and R3 ( ${ }^{+} p<0.05$ compared to non-stressed controls on the same stage). White bars: unstressed controls; gray bars: CUS. significant differences between treatment groups in trials to criterion for the SD task on the training day $\left(\mathrm{F}_{1,39}=0.526\right.$, $p=0.47$ for Stress; $F_{2,39}=0.902, p=0.41$ for drug).

For performance on the test day, three-way ANOVA (stress $\times$ drug $\times$ task) revealed a significant main effect of task $\left(\mathrm{F}_{6,234}=14.77, p<0.001\right)$, but not drug $\left(\mathrm{F}_{2,39}=\right.$ $1.38, p=0.26)$ or stress $\left(\mathrm{F}_{1,39}=0.42, p=0.52\right)$. However, there was a significant $\operatorname{drug} \times$ stress $\times$ task interaction $\left(\mathrm{F}_{12,234}=1.89, p<0.05\right)$. As in Experiment 1 , post hoc analysis of the task effect across groups showed the trials required to reach criterion on $\mathrm{R} 1, \mathrm{R} 3$, and $\mathrm{ED}$ to be significantly higher than on the other tasks (Figure 3). Subsequent post hoc ANOVA for all task stages found a significant stress $\times$ drug interaction only for the ED stage $\left(\mathrm{F}_{2,39}=6.746, p<0.003\right.$; Figure 4$)$. No other main effects or interactions were revealed on any other test stages. Post hoc planned comparisons for the ED stage showed that in vehicle-treated rats, as in experiment 1 , CUS induced a significant performance deficit relative to unstressed controls (Figure 4). Chronic treatment with both DMI and SCIT prevented the CUS-induced deficit in ED cognitive setshifting. In addition, in non-stressed rats, chronic treatment with SCIT alone, but not DMI, impaired ED set shifting relative to vehicle-treated controls.

With respect to effects on the elevated plus-maze, ANOVA revealed a significant main effect of stress on OTR for both time $\left(\mathrm{F}_{1,45}=10.63, p<0.005\right)$ and entries $\left(\mathrm{F}_{1,45}=6.35, \quad p<0.05\right)$, but no main effect of drug $\left(\mathrm{F}_{2,45}=0.5, p=0.61\right.$ and $\mathrm{F}_{2,45}=0.38, p=0.68$, respectively). In replication of the effect seen in experiment 1 , CUS induced an anxiogenic effect in vehicle-treated rats, indicated by a significant reduction in OTR for time $(p<0.05$, Figure 5$)$. The reduction in OTR for entries, although of comparable magnitude to that seen in experiment 1 , did not achieve significance by post hoc comparison. In non-stressed rats, both drugs alone appeared to have modest, though non-significant anxiogenic effects, most notably for SCIT (Figure 5). Most importantly, in
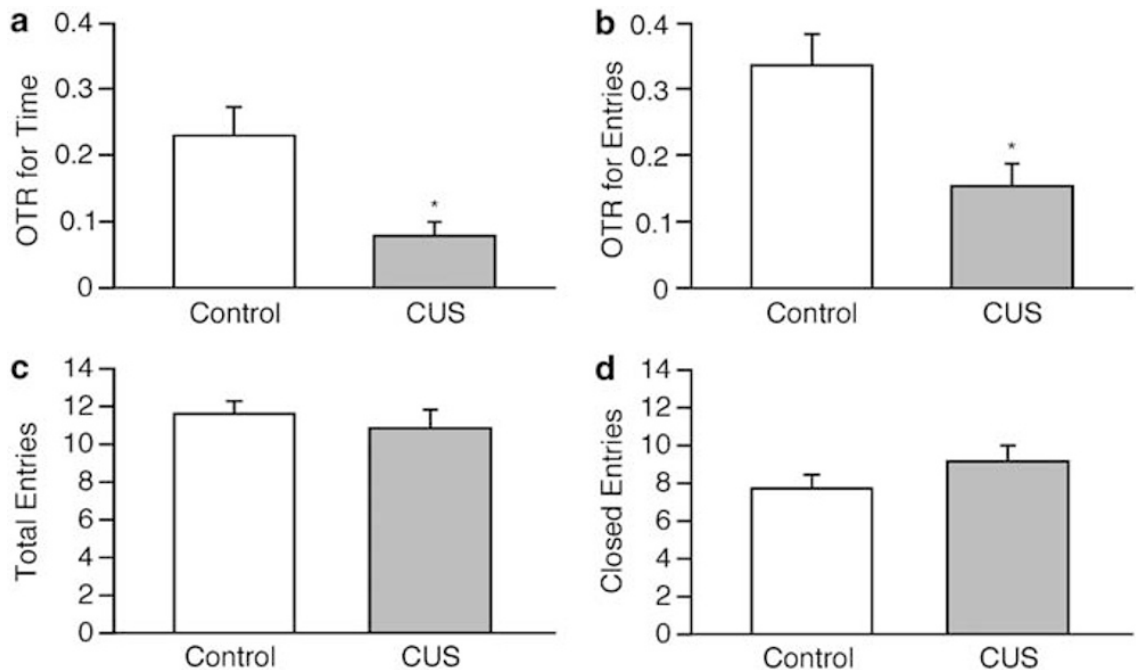

Figure 2 Effect of CUS on anxiety-like behavior on the elevated plus-maze. Rats subjected to CUS displayed an increase in anxiety-like behavior, seen as a reduction in open-arm exploration compared to controls, measured as OTR for time (a) or entries (b) $(* p<0.005$, data expressed as mean \pm SEM, $n=16-$ 17/group). The CUS-induced reduction in open-arm exploration was not attributable to reduced exploratory drive or locomotor activity, as there was no difference between groups in total number of arm entries (c) or number of closed-arm entries (d). 


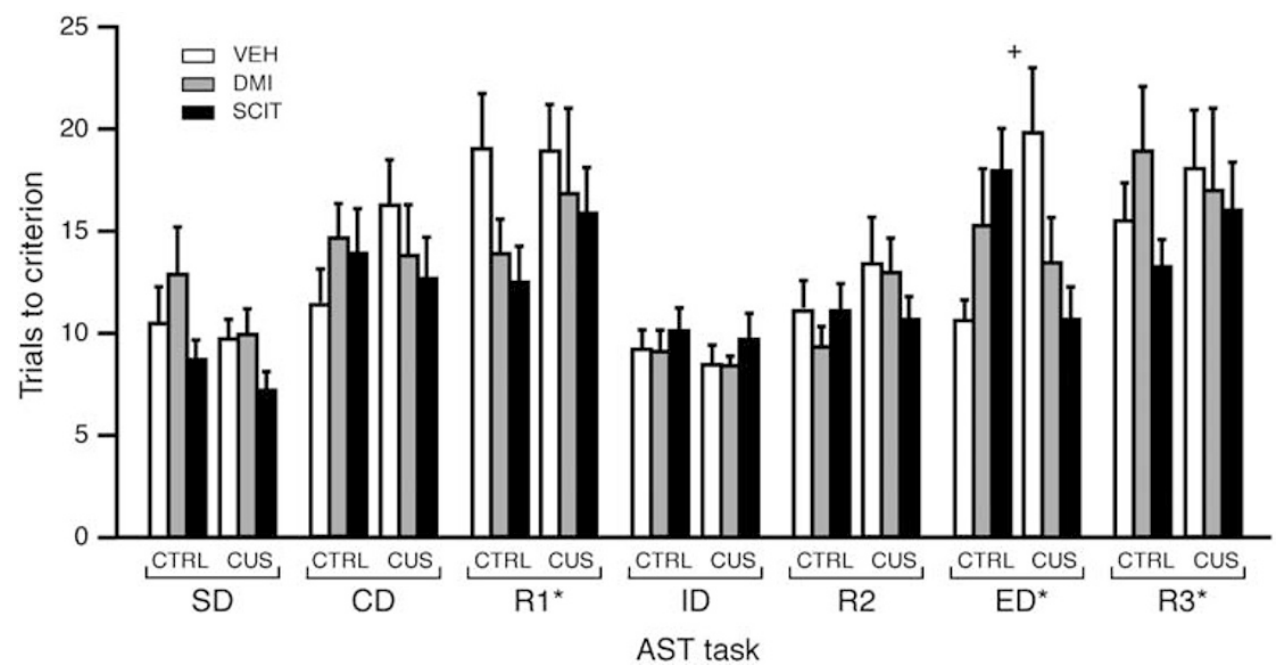

Figure 3 Effects of chronic administration of DMI $(7.5 \mathrm{mg} / \mathrm{kg} /$ day $)$ or SCIT (I $\mathrm{mg} / \mathrm{kg} /$ day) treatment during CUS on attentional set-shifting performance. As in experiment I, significantly more trials were required to reach criterion on the RI, ED and R3 tasks (Main effect of Stage: * $p<0.01$ compared to the other stages, collapsed across treatment groups). There was also a significant drug $\times$ stress $\times$ task interaction $(p<0.05)$. Post hoc ANOVAs for each stage revealed a significant stress $x$ drug interaction only for the ED stage $(+p<0.05)$. For clarity, subsequent post hoc analysis of this interaction effect on ED performance is illustrated in more detail in Figure 4. All data are expressed as mean \pm SEM, $n=6-8$ /group.

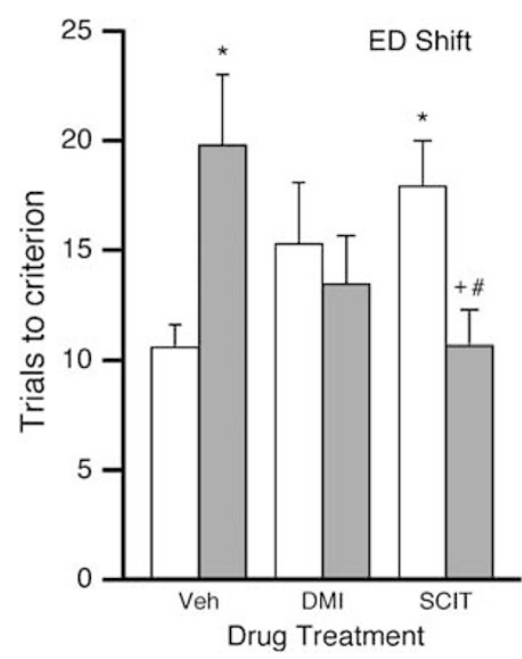

Figure 4 Effects of chronic treatment with DMI $(7.5 \mathrm{mg} / \mathrm{kg} / \mathrm{day})$ or SCIT $(10 \mathrm{mg} / \mathrm{kg} / \mathrm{day})$ on the CUS-induced alterations specifically on ED setshifting performance. In vehicle-treated rats, as in experiment I, CUS induced a significant deficit in ED set shifting. This effect was prevented by chronic treatment with either DMI or SCIT. Treatment with SCIT alone produced an increase in trials to criterion in unstressed rats $(* p<0.005$ compared to vehicle-treated unstressed controls; ${ }^{+} p<0.05$ compared to vehicle-treated rats subjected to CUS; ${ }^{\#} p<0.01$ compared to SCITtreated, unstressed controls; data expressed as mean \pm SEM, $n=6-8$ / group). White bars: unstressed controls; gray bars: CUS.

stressed animals, chronic treatment with DMI prevented the CUS-induced reduction in open arm exploration on the plus maze (Figure 5a and b). The effect of SCIT was less clear; in SCIT-treated groups, CUS still caused a decrease in openarm exploration comparable to that seen in vehicle-treated groups, but this was not a significant difference, possibly because of the reduction in baseline OTR caused by SCIT treatment alone in unstressed animals (see Figure $5 \mathrm{a}$ and $\mathrm{b}$ ).

There was a significant effect of drug on both total entries $\left(\mathrm{F}_{2,45}=4.41, p<0.05\right)$ and closed arm entries $\left(\mathrm{F}_{2,45}=4.35\right.$, $p<0.05)$, but no main effect of stress $\left(\mathrm{F}_{1,45}=1.96, p=0.17\right.$ and $\mathrm{F}_{1,45}=0.002, p=0.97$, respectively). Although no individual comparison were significant by post-hoc analysis, this effect was apparently due to a modest reduction in locomotor activity seen only in rats subjected to CUS and treated chronically with DMI (Figure $5 c, d$ ).

Analysis of the effects of CUS and drug treatment on body weight gain over time demonstrated significant main effects of stress $\left(\mathrm{F}_{1,45}=14.77, p<0.001\right)$ and time $\left(\mathrm{F}_{5,225}=37.64\right.$, $p<0.001)$, and an effect of drug that approached but did not achieve significance $\left(\mathrm{F}_{2,45}=2.89, p=0.066\right)$. CUS significantly reduced body weight gain across drug treatment groups (5-10\% difference between CUS and unstressed rats within drug treatment groups, Figure 6), similar in magnitude to effects reported previously (Willner et al, 1996). There was a similar and parallel decrease in body weight in all groups during the week before AST testing, during which rats were subjected to food restriction (a decrease of $4-6 \%$ between days 10-17 of CUS treatment, Figure 6). Finally, rats treated chronically with SCIT tended to have higher body weight than their vehicle-treated counterparts in both the CUS- and non-stressed conditions (Figure 6).

\section{DISCUSSION}

In this study, we found that CUS induced a cognitive deficit, manifest most consistently across both experiments as an increase in the number of trials to criterion in the extradimensional cognitive set shifting task. Along with the cognitive deficit, rats subjected to CUS also consistently displayed increased anxiety-like behavior on the elevated plus-maze. Further, we found that the cognitive performance deficit in attentional set shifting induced by CUS was prevented by chronic treatment with antidepressant drugs of two different classes, an SSRI (SCIT) and a selective NRI (desipramine). Chronic treatment with DMI also prevented 

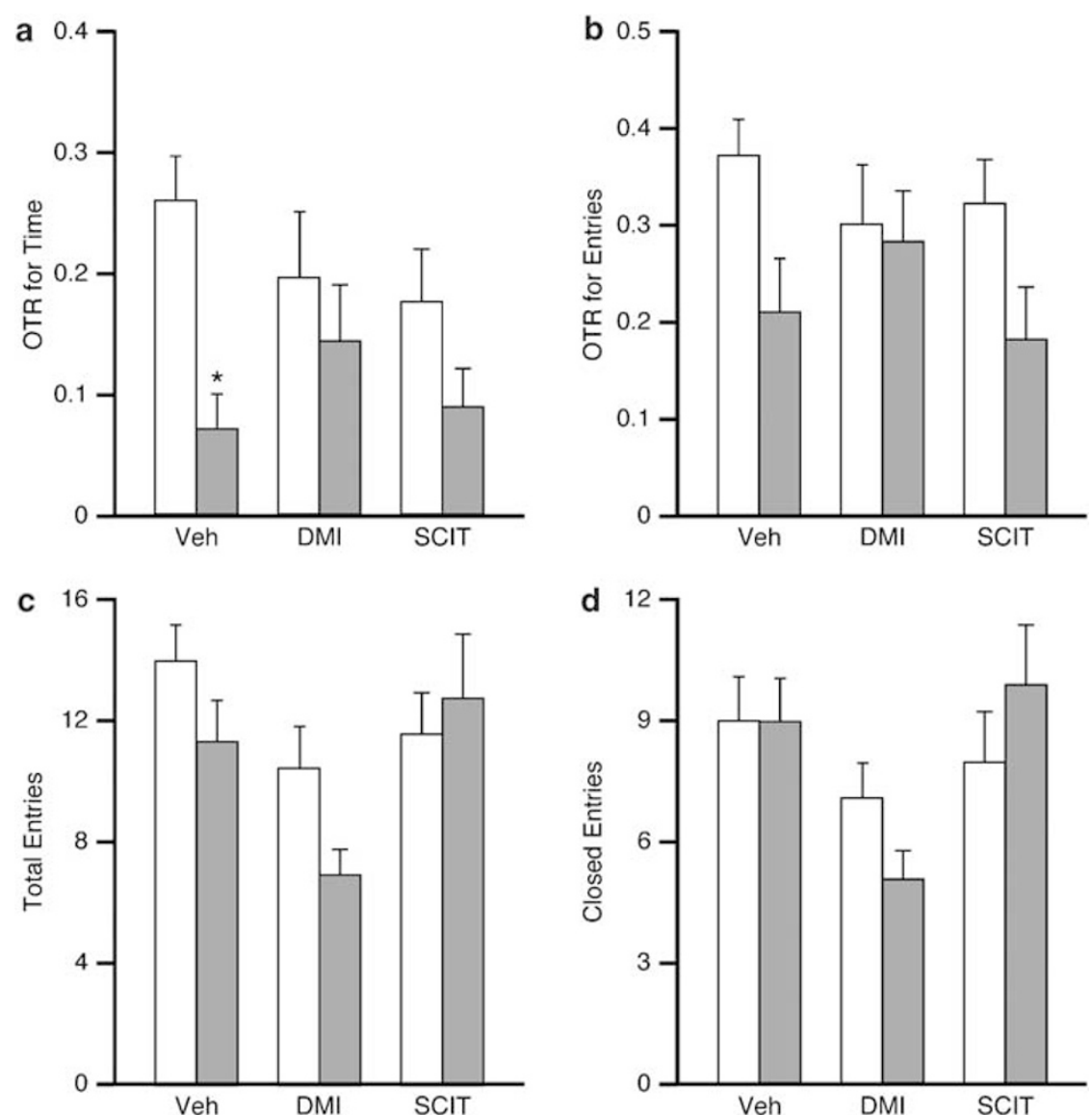

Figure 5 Effects of chronic treatment with DMI $(7.5 \mathrm{mg} / \mathrm{kg} /$ day $)$ or SCIT $(10 \mathrm{mg} / \mathrm{kg} /$ day $)$ on the CUS-induced alterations in anxiety-like behavior on the elevated plus-maze. As in experiment I, CUS caused a reduction in open-arm exploration, seen as a significant decrease in OTR for time (a: * $<0.05$ compared to vehicle-treated unstressed controls). The CUS-induced reduction in OTR for entries was comparable to that in experiment I, but did not achieve significance by post hoc analysis (b). In addition, there was a slight reduction in locomotor activity, measured by total entries or closed-arm entries, seen only in DMI-treated rats subjected to CUS (c and d) (data are expressed as mean \pm SEM $n=8-9 /$ group). White bars: unstressed controls; gray bars: CUS.

the CUS-induced increase in anxiety-like behavior on the plus-maze.

Major depression and anxiety disorders are associated with functional and morphological alterations in the prefrontal cortex and limbic system, along with symptoms reflecting, among other things, both cognitive dysfunction and anxiety (Brown et al, 1998; Mineka et al, 1998). Clinical studies have shown that stressful life experiences are important etiological factors in the development and maintenance of depression and affective disorders (Kendler et al, 1995, 1998; Kessler, 1997), particularly associated with cognitive and emotional biases (Beck, 1976; Beck et al, 1987; Coles and Heimberg, 2002; Mathews and Mackintosh, 1998). In the present study, CUS induced cognitive impairments in the AST on the stimulus reversal trials (experiment 1) and on the ED set-shifting task (replicated in both experiments). The ED set-shifting task in particular is a measure of cognitive flexibility. Subjects must utilize feedback from their environment to modify established patterns of behavior, recognizing that a previously learned set of rules is no longer valid, inhibiting behavioral choices based on those rules, then learning a new set of rules based on a new pattern of reinforcement (Birrell and Brown, 2000; Chen et al, 2004; Lapiz and Morilak, 2006). This process of cognitive flexibility depends on the mPFC in rats, and the analogous dorsolateral PFC in primates, as lesions in these regions specifically impaired performance on ED setshifting (Birrell and Brown, 2000; Dias et al, 1996).

Deficits in such cognitive flexibility are detected on the WCST in human patients with dorsolateral prefrontal cortical lesions (Stuss et al, 2000), and with a variety of neuropsychiatric disorders that involve deficits of mPFC functioning (Fossati et al, 1999; Goldstein et al, 2002). Further, deficits in cognitive flexibility, resulting in perseverative perceptual, emotional, and memory biases have been suggested to be important in depression and anxiety disorders (Beck, 1976; Beck et al, 1987; Coles and Heimberg, 2002; Mathews and Mackintosh, 1998).

The deficit in ED set shifting seen in the present study after CUS is consistent with a recent report (Liston et al, 2006), published as the current work was in preparation, in which a similar deficit in attentional set shifting was seen following repeated restraint stress, a model proposed to induce some of the pathological changes underlying affective disorders (McEwen, 2003; Reagan et al, 2004). In addition to the cognitive deficit, Liston et al (2006) also reported that repeated restraint stress induced apical dendritic atrophy of prefrontal cortical pyramidal neurons, in replication of previous studies (Radley et al, 2004, 2006). In these studies, dendritic atrophy and spine retraction 


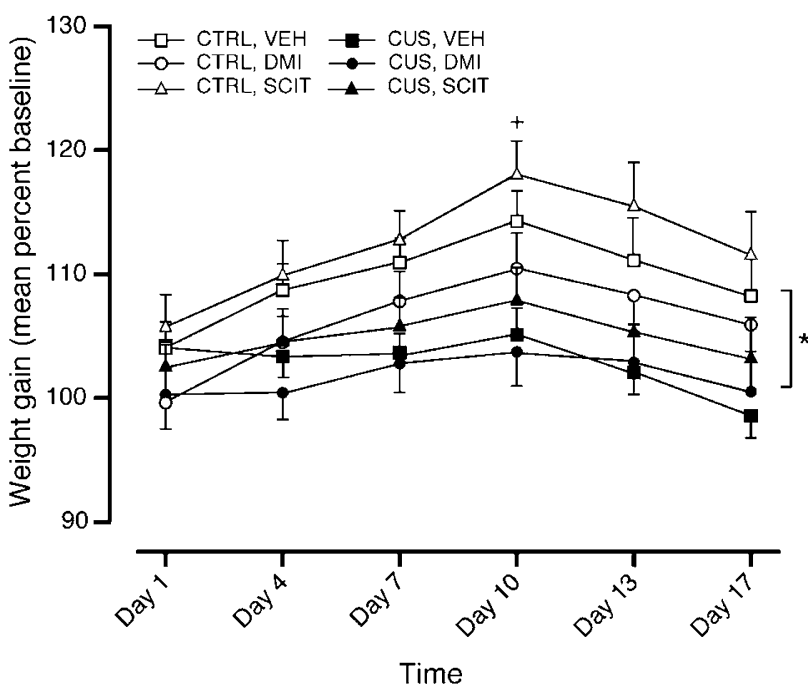

Figure 6 Body weight gain over the course of CUS treatment and behavioral testing. CUS reduced the rate of body weight gain over the course of treatment, resulting in a 5-10\% difference in weight gain in each drug group $(* p<0.00$ I CUS compared to non-stressed rats across drug groups). There was a similarly modest (4-6\%) and parallel decrease in body weight in all groups during the week before AST testing, during which rats were subjected to food restriction $\left({ }^{+} p<0.001\right.$ day 10 compared to days 1 and 17 across groups). Data are expressed as mean percent of pretreatment starting weight \pm SEM ( $n=8-9 /$ group).

following chronic restraint stress was restricted to the most apical dendrites of the pyramidal cells, where monoaminergic neurotransmission, specifically $5 \mathrm{HT}$ acting at 5HT2A receptors and NE acting at $\alpha_{1}$-adrenergic receptors, induces excitatory potentials in pyramidal cells through facilitation of glutamatergic input from thalamocortical afferents (Marek and Aghajanian, 1999). Thus, in the present study, it is possible that CUS may have induced monoaminergic facilitation of stress-related glutamate neurotransmission, which in turn may have led to excitotoxic damage in the apical dendritic field of cortical pyramidal cells, resulting in the observed cognitive deficit. A potential role for monoaminergic facilitation in the mechanisms underlying the cognitive deficit is currently under investigation.

However, even if monoamine-related mechanisms are not involved directly in the cellular pathology that develops in mPFC after chronic stress, such a localized loss of synapses in a region of the pyramidal cell dendritic tree that is normally modulated by monoaminergic afferents may explain in part the deficit of cognitive functioning, and specifically the deficit in ED set shifting induced by CUS. We have shown previously that ED set shifting was enhanced by elevating noradrenergic tone in mPFC, through activation of $\alpha_{1}$-adrenergic receptors (Lapiz and Morilak, 2006). In another study, ED set-shifting was disrupted specifically by selective deafferentiation of $\mathrm{NE}$ terminals in rat mPFC (Eichenbaum et al, 2003). Thus, a chronic stress-induced loss of the pyramidal cell dendrites and synapses that are normally targeted by noradrenergic facilitatory inputs may account for the decrement observed in cognitive performance following CUS.

Serotonin also facilitates glutamate transmission at pyramidal cell dendrites in MPFC (Marek and Aghajanian,
1999), but the potential influence of serotonin on cognition is less clear. Destruction of cortical serotonergic projections or depletion of 5-HT produced deficits in reversal learning, rather than cognitive set shifting (Clarke et al, 2005, 2007; Nomura, 1992). Reversal learning appears to be related more to the function of orbitofrontal cortex rather than medial prefrontal cortex (Clarke et al, 2007; McAlonan and Brown, 2003). In the present study, this may account in part for the inconsistent effects of CUS seen on stimulus reversal trials . It has been suggested that reversal of spatial learning in the Morris water maze may be impaired by CUS (Hill et al, 2005). However, by contrast with the effects on ED set shifting and morphologic changes seen in mPFC after repeated restraint stress, Liston et al (2006) reported no effects on reversal learning and no morphological changes in orbitofrontal cortex. Thus, it is possible that the CUS treatment in the present study may have induced inconsistent and/or modest changes in endogenous serotonergic activity specifically in orbitofrontal cortex.

However, elevating serotonergic function pharmacologically by chronic treatment with SSRIs improved the dysregulated cerebral blood flow and metabolic activity seen in mPFC of depressed subjects (Kennedy et al, 2001), consistent with the observation that SCIT prevented the CUS-induced deficit in ED set shifting in the present study. Thus, there must be some convergence of effect of SSRI and selective NRI treatment in improving mPFC function. One possible mechanism may involve the demonstrated interactions between the serotonergic and noradrenergic systems, as chronic administration of either SSRI or selective NRI drugs modulates the activity of both (Szabo and Blier, 2001; Szabo et al, 2000). In addition, both SSRI and selective NRI treatment may increase dopamine neurotransmission (reviewed in Morilak and Frazer, 2004), and dopamine has also been shown to enhance ED set shifting in mPFC (Ragozzino, 2002; Tunbridge et al, 2004). An increase in dopaminergic function might also contribute to the beneficial effect of chronic antidepressant treatment on measures of anhedonia induced by chronic mild stress (Bonhomme and Esposito, 1998; Willner, 1997; Willner et al, 1994). Moreover, the dopamine agonist, apomorphine, also increased open arm exploration on the elevated plusmaze, an effect that was attenuated by D2 dopamine receptor blockade (Garcia et al, 2005).

In addition to the cognitive deficit, CUS exposure also produced a consistent increase in anxiety-like behavior on the elevated-plus maze, seen as a decrease in open-arm exploration. As in our own previous work and that of others (eg, Griebel et al, 1997; Hata et al, 2001; Khoshbouei et al, 2002), OTR for time was found to be the more consistent and reliable measure of open-arm exploration, and a more sensitive indicator of anxiogenic response to CUS, but similar effects were also seen with OTR for Entries. This combined effect of a decrease in cognitive capability, in particular cognitive flexibility, together with a basal state of anxiety-like behavior induced by CUS is consistent with the changes in cognition and anxiety seen in major depressive disorder. Indeed, anxiety is a prominent component of depression, and anxiety disorders are frequently comorbid with major depression (Gorman, 1996/1997; Mineka et al, 1998). Thus, understanding the mechanisms by which CUS induced both a cognitive deficit on the AST and anxiety-like 
behavior on the elevated plus-maze may help us to better understand the mechanisms by which chronic or severe life stress may contribute to the development of both depression and anxiety disorders.

By contrast with the prefrontal cortex, where repeated stress induced dendritic atrophy, the opposite response, including dendritic hypertrophy and increased dendritic elaboration has been observed in the extended amygdala, specifically in the bed nucleus of the stria terminalis (Vyas et al, 2003). This may explain the increase in stress-induced anxiety-like behavior on the elevated plus-maze, as we and others have shown such behavioral responses to involve the extended amygdala, in particular the lateral bed nucleus of the stria terminalis (Cecchi et al, 2002a; Davis and Shi, 1999). Further, behavioral reactivity on this test was shown to be facilitated specifically by an acute stress-evoked increase in the release of $\mathrm{NE}$, rather than by basal noradrenergic tone (Cecchi et al, 2002a). Thus, chronic NE reuptake blockade may have attenuated the anxiety-like behavioral activity observed on the plus-maze following CUS by reducing phasic stress-evoked noradrenergic reactivity (Szabo and Blier, 2001; Szabo et al, 2000; Valentino et al, 1990).

The results of numerous preclinical experiments testing the effects of 5-HT-related drugs in general, and SSRIs in particular, in various animal models of anxiety have been equivocal at best (see Griebel, 1995; Griebel et al, 1997; Handley, 1995). In the elevated plus-maze and related tests (eg, social interaction), both acute and chronic treatment with SSRIs have typically had no effect, or have had anxiogenic effects (File et al, 1999; Silva and Brandão, 2000). Thus, it is perhaps not surprising that chronic SCIT treatment did not prevent the CUS-induced increase in anxiety-like behavior on the plus-maze in the present study, nor that SCIT treatment alone had a modest anxiogenic effect. As SSRIs are clearly effective in treating human anxiety, one obvious implication from this literature, as well as the present study, is that many commonly used assays of anxiety-like behavior in rats are not valid models of human anxiety disorders that are responsive to SSRI treatment (eg, see Handley, 1995; Handley and McBlane, 1993).

A more subtle interpretation, however, is that the anxiolytic effects of chronic SSRI treatment may in fact be attributable more to their effects on cognitive or perceptual processes that might lead to a disproportionate or inappropriate subjective feeling of distress and anxiety in the absence of an appropriately threatening stimulus (see Beck, 1976; Coles and Heimberg, 2002; Mathews and Mackintosh, 1998), rather than any effect they may exert on the presumably appropriate and adaptive behavioral responses to acute stressors that are measured in most animal models of 'anxiety' (Handley and McBlane, 1993). It would thus be useful to be able to adopt an animal model of anxiety in which the behavioral response was not adaptive (as it is in such models as the elevated plus-maze, defensive burying or social interaction tests), but rather was seen as pathological and inappropriate to the context. If such a model could be developed, which might include a perceptual or interpretive cognitive component, it might better detect the anxiolytic effects of SSRIs than those models of anxiety-like behavior currently used.

In the present study, antidepressants were given before and during the CUS treatment. For many patients, depression becomes a chronic condition with relapse occurring in over half (Keller and Boland, 1998). Long-term preventive maintenance of antidepressant treatment reduces the chance of recurrence by two-thirds (Geddes et al, 2003). Approximately $60 \%$ of such patients are at risk of experiencing a recurrent episode of depression within a year if left untreated, whereas those who continue antidepressant treatment have a recurrence rate of only $10-30 \%$ (see Hirschfeld, 2001). This beneficial treatment effect is similar across different classes of antidepressants, including SSRIs and selective NRIs. Thus, it is important to consider the mechanisms underlying the potentially beneficial effects of prophylactic antidepressant treatment for recurrent depression. In the present study, chronic treatment with either DMI or SCIT prevented the CUS-induced cognitive deficit in ED set-shifting, and chronic treatment with DMI also prevented the stress-induced anxiety-like reduction in open-arm exploration on the EPM. It is possible, then, that the beneficial effects of long-term maintenance therapy are related to the beneficial effects of antidepressant drugs on cognitive function and/or state anxiety.

In conclusion, these results show that CUS produces a cognitive deficit in rats similar to that seen in patients with major depressive disorder, as well as a state of anxiety that also occurs in depression. Furthermore, pretreatment with a representative drug from the selective NRI class of antidepressants prevented both the cognitive and anxietylike behavioral alterations caused by CUS. Pretreatment with a representative SSRI prevented the cognitive deficit, but its effect on anxiety was, perhaps surprisingly, less clear. The CUS model, therefore, should prove useful for further investigation of the neurobiological mechanisms underlying cognitive and behavioral consequences of chronic stress exposure that may be important in the development of depression and anxiety disorders, as well as mechanisms that may account for the beneficial effects of chronic antidepressant drug treatment for stress-related psychiatric disorders

\section{ACKNOWLEDGEMENTS}

We thank Dr Danet Lapiz and Dr Xin-Yun Lu, Department of Pharmacology, UTHSCSA for their assistance and advice with the attentional set-shifting procedure and the CUS procedure, respectively. Support provided by research grants from the National Institutes of Health (MH53851, MH72672 and MH57001). Gus Rodriguez was a summer undergraduate research fellow supported by a NIH Mental Health Research Gateway Program grant (MH065504).

\section{STATEMENT OF DISCLOSURE AND CONFLICT OF INTEREST}

The authors have no conflicts of interest to report, nor any involvement to disclose, financial or otherwise, that may bias the conduct, interpretation or presentation of this work. Dr Frazer has received support from Forest Laboratories for a study of SCIT that is unrelated to the current work. Organizations from whom the authors have received compensation for professional services. Corina O. Bondi: none; Gus Rodriguez: none; Georgianna Gould: none; Alan Frazer: Advisory boards: Eli Lilly and Co., H. 
Lundbeck A/S, Cyberonics, Inc. Grant Support: Eli Lilly and Co., Forest Labs, Cyberonics, Inc., Servier; David Morilak: Advisory boards: Eli Lilly and Co., Sanofi-Aventis Grant Support: Servier.

\section{REFERENCES}

Anisman H, Matheson K (2005). Stress, depression, and anhedonia: Caveats concerning animal models. Neurosci Biobehav Revs 29: $525-546$.

Austin M-P, Mitchell P, Goodwin GM (2001). Cognitive deficits in depression: possible implications for functional neuropathology. Br J Psychiatry 178: 200-206.

Beats BC, Sahakian BJ, Levy R (1996). Cognitive performance in tests sensitive to frontal lobe dysfunction in the elderly depressed. Psychol Med 26: 591-604.

Beck AT (1976). Cognitive Therapy and the Emotional Disorders. Int. Univ. Press: New York.

Beck AT, Brown G, Steer RA, Eidelson JI, Riskind JH (1987), Differentiating anxiety and depression: A test of the cognitive content-specificity hypothesis. J Abnormal Psychol 96: 179-183.

Birrell JM, Brown VJ (2000). Medial frontal cortex mediates perceptual attentional set shifting in the rat. J Neurosci 20: $4320-4324$

Bondi CO, Gould G, Lu X-Y, Frazer A, Morilak DA (2006). Chronic unpredictable stress induces a cognitive deficit and anxiety-like behavior in rats. Soc Neurosci Abstr 32 Online: Program no. 155.8.

Bonhomme N, Esposito E (1998). Involvement of serotonin and dopamine in the mechanism of action of novel antidepressant drugs: A review. J Clin Pharmacol 18: 447-454.

Brown TA, Chorpita BF, Barlow DH (1998). Structural relationships among dimensions of the DSM-IV anxiety and mood disorders and dimensions of negative affect, positive affect, and autonomic arousal. J Abnormal Psychol 107: 179-192.

Cecchi M, Khoshbouei H, Javors M, Morilak DA (2002a). Modulatory effects of norepinephrine in the lateral bed nucleus of the stria terminalis on behavioral and neuroendocrine responses to acute stress. Neuroscience 112: 13-21.

Cecchi M, Khoshbouei H, Morilak DA (2002b). Modulatory effects of norepinephrine, acting on $\alpha_{1}$-receptors in the central nucleus of the amygdala, on behavioral and neuroendocrine responses to acute immobilization stress. Neuropharmacology 43: 1139-1147.

Chen KC, Baxter MG, Rodefer JS (2004). Central blockade of muscarinic cholinergic receptors disrupts affective and attentional set-shifting. Eur J Neurosci 20: 1081-1088.

Clarke HF, Walker SC, Crofts HS, Dalley JW, Robbins TW, Roberts AC (2005). Prefrontal serotonin depletion affects reversal learning but not attentional set shifting. J Neurosci 25: 532-538.

Clarke HF, Walker SC, Dalley JW, Robbins TW, Roberts AC (2007). Cognitive inflexibility after prefrontal serotonin depletion is behaviorally and neurochemically specific. Cerebral Cortex 17: 18-27.

Coles ME, Heimberg RG (2002). Memory biases in the anxiety disorders: current status. Clin Psychol Rev 22: 587-627.

Davis M, Shi C (1999). The extended amygdala: are the central nucleus of the amygdala and the bed nucleus of the stria terminalis differrentially involved in fear versus anxiety? Ann NY Acad Sci 877: 281-291.

Dias R, Robbins TW, Roberts AC (1996). Primate analogue of the Wisconsin Card Sorting Test: effects of excitotoxic lesions of the prefrontal cortex of the marmoset. Behav Neurosci 110: $872-886$.

Eichenbaum HB, Ross R, Raji A, McGaughy JA (2003). Noradrenergic, but not cholinergic, deafferentation of the infralimbic/ prelimbic cortex impairs attentional set-shifting. Soc Neurosci Abstr 29 Online: Program no. 940.7.
File SE, Ouagazzal AM, Gonzalez LE, Overstreet DH (1999). Chronic fluoxetine in tests of anxiety in rat lines selectively bred for differential 5-HT1A receptor function. Pharmacol Biochem Behav 62: 695-701.

Fossati P, Amar G, Raoux N, Ergis AM, Allilaire JF (1999). Executive functioning and verbal memory in young patients with unipolar depression and schizophrenia. Psychiatry Res 89: 171-187.

Garcia AM, Martinez R, Brandão ML, Morato S (2005). Effects of apomorphine on rat behavior in the elevated plus-maze. Physiol Behav 85: 440-447.

Geddes JR, Carney SM, Davies C, Furukawa TA, Kupfer DJ, Frank $\mathrm{E}$ et al (2003). Relapse prevention with antidepressant drug treatment in depressive disorders: a systematic review. Lancet 361: 653-661.

Goldstein G, Minshew NJ, Allen DN, Seaton BE (2002). Highfunctioning autism and schizophrenia: a comparison of an early and late onset neurodevelopmental disorder. Arch Clin Neuropsychol 17: 461-475.

Gorman JM (1996/1997). Comorbid depression and anxiety spectrum disorders. Depress Anxiety 4: 160-168.

Griebel G (1995). 5-Hydroxytryptamine-interacting drugs in animal models of anxiety disorders: more than 30 years of research. Pharmac Ther 65: 319-395.

Griebel G, Rodgers RJ, Perrault G, Sanger DJ (1997). Risk assessment behaviour: evaluation of utility in the study of 5-HT-related drugs in the rat elevated plus-maze test. Pharm Biochem Behav 57: 817-827.

Handley SL (1995). 5-Hydroxytryptamine pathways in anxiety and its treatment. Pharmac Ther 66: 103-148.

Handley SL, McBlane JW (1993). 5HT drugs in animal models of anxiety. Psychopharmacology 112: 13-20.

Hata T, Nishikawa H, Itoh E, Funakami Y (2001). Anxiety-like behavior in elevated plus-maze tests in repeatedly cold-stressed mice. Jpn J Pharmacol 85: 189-196.

Hill MN, Patel S, Carrier EJ, Rademacher DJ, Ormerod BK, Hillard CJ et al (2005). Downregulation of endocannabinoid signaling in the hippocampus following chronic unpredictable stress. Neuropsychopharmacology 30: 508-515.

Hirschfeld RM (2001). Clinical importance of long-term antidepressant treatment. Br J Psychiatry 42(Suppl): S4-S8.

Katz RJ, Roth KA, Carroll BJ (1981). Acute and chronic effects on open field activity in the rat: implications for a model of depression. Neurosci Biobehav Revs 5: 247-251.

Keller MB, Boland RJ (1998). Implications of failing to achieve successful long-term maintenance treatment of recurrent unipolar major depression. Biol Psychiatry 44: 348-360.

Kendler KS, Karkowski LM, Prescott CA (1998). Stressful life events and major depression: risk period, long-term contextual threat, and diagnostic specificity. J Nerv Mental Dis 186: 661-669.

Kendler KS, Karkowski LM, Prescott CA (1999). Causal relationship between stressful life events and the onset of major depression. Am J Psychiatry 156: 837-841.

Kendler KS, Kessler RC, Walters EE, MacLean C, Neale MC, Heath AC et al (1995). Stressful life events, genetic liability, and onset of an episode of major depression in women. Am J Psychiatry 152: 833-842.

Kennedy SH, Evans KR, Kruger S, Mayberg HS, Meyer JH, McCann $S$ et al (2001). Changes in regional brain glucose metabolism measured with positron emission tomography after paroxetine treatment of major depression. Am J Psychiatry 158: 899-905.

Kessler RC (1997). The effects of stressful life events on depression. Ann Rev Psychol 48: 191-214.

Khoshbouei H, Cecchi M, Dove S, Javors M, Morilak DA (2002). Behavioral reactivity to stress: amplification of stress-induced noradrenergic activation elicits a galanin-mediated anxiolytic effect in central amygdala. Pharmacol Biochem Behav 71: 407-417. 
Lapiz MDS, Morilak DA (2006). Noradrenergic modulation of cognitive function in rat medial prefrontal cortex as measured by attentional set shifting capability. Neuroscience 137: 1039-1049.

Liston C, Miller MM, Goldwater DS, Radley JJ, Rocher AB, Hof PR et al (2006). Stress-induced alterations in prefrontal cortical dendritic morphology predict selective impairments in perceptual attentional set-shifting. J Neurosci 26: 7870-7874.

Lu X-Y, Kim CS, Frazer A, Zhang W (2006). Leptin: a potential novel antidepressant. Proc Natl Acad Sci USA 103: 1593-1598.

Marek GJ, Aghajanian GK (1999). 5- $\mathrm{HT}_{2 \mathrm{~A}}$ receptor or $\alpha_{1}$ adrenoceptor activation induces excitatory postsynaptic currents in layer V pyramidal cells of the medial prefrontal cortex. Eur J Pharmacol 367: 197-206.

Mathews A, Mackintosh B (1998). A cognitive model of selective processing in anxiety. Cognitive Ther Res 22: 539-560.

McAlonan K, Brown VJ (2003). Orbital prefrontal cortex mediates reversal learning and not attentional set shifting in the rat. Behav Brain Res 146: 97-103.

McEwen BS (2003). Mood disorders and allostatic load. Biol Psychiatry 54: 200-207.

Merriam EP, Thase ME, Haas GL, Keshavan MS, Sweeney JA (1999). Prefrontal cortical dysfunction in depression determined by Wisconsin Card Sorting Test performance. Am J Psychiatry 156: $780-782$.

Miller E, Lewis P (1977). Recognition memory in elderly patients with depression and dementia: a signal detection analysis. J Abnormal Psychol 86: 84-86.

Mineka S, Watson D, Clark LA (1998). Comorbidity of anxiety and unipolar mood disorders. Ann Rev Psychol 49: 377-412.

Morilak DA, Frazer A (2004). Antidepressants and brain monoaminergic systems: a dimensional approach to understanding their behavioral effects in depression and anxiety disorders. Int $J$ Neuropsychopharmacol 7: 193-218.

Murphy FC, Sahakian BJ, Rubinsztein JS, Michael A, Rogers RD, Robbins TW et al (1999). Emotional bias and inhibitory control processes in mania and depression. Psychol Med 29: 1307-1321.

Nikisch G, Mathé AA, Czernik A, Eap CB, Jiménez-Vasquez P, Brawand-Amey M, Baumann P (2004). Stereoselective metabolism of citalopram in plasma and cerebrospinal fluid of depressive patients: relationship with 5-HIAA in CSF and clinical response. J Clin Psychopharmacol 24: 283-290.

Nikulina EM, Miczek KA, Hammer RP (2005). Prolonged effects of repeated social defeat stress on mRNA expression and function of $\mu$-opioid receptors in the ventral tegmental area of rats. Neuropsychopharmacology 30: 1096-1103.

Nomura M (1992). Effects of bifemelane on discrimination learning of serotonergic-dysfunction rats. Pharm Biochem Behav 42: 721-731.

Pardon M-C, Gould GG, Garcia A, Phillips L, Cook MC, Miller SA et al (2002). Stress reactivity of the brain noradrenergic system in three rat strains differing in their neuroendocrine and behavioral responses to stress: implications for susceptibility to stress-related neuropsychiatric disorders. Neuroscience 115: 229-242.

Perry PJ, Zeilmann C, Arndt S (1994). Tricyclic antidepressant concentrations in plasma: an estimate of their sensitivity and specificity as a predictor of response. J Clin Psychopharmacol 14: 230-240.

Prasko J, Hornacek J, Zalesky R, Kopecek M, Novak T, Paskova B et al (2004). The change of regional brain metabolism (18FDG PET) in panic disorder during the treatment with cognitive behavioral therapy or antidepressants. Neuro Endocrinol Lett 25: 340-348.

Purcell R, Maruff P, Kyrios M, Pantelis C (1997). Neuropsychological function in young patients with unipolar major depression. Psychol Med 27: 1277-1285.

Radley JJ, Rocher AB, Miller M, Janssen WGM, Liston C, Hof PR et al (2006). Repeated stress induces dendritic spine loss in the rat medial prefrontal cortex. Cerebral Cortex 16: 313-320.

Radley JJ, Sisti HM, Hao J, Rocher AB, McCall T, Hof PR et al (2004). Chronic behavioral stress induces apical dendritic reorganization in pyramidal neurons of the medial prefrontal cortex. Neuroscience 125: 1-6.

Ragozzino ME (2002). The effects of dopamine D(1) receptor blockade in the prelimbic-infralimbic areas on behavioral flexibility. Learning Memory 9: 18-28.

Reagan LP, Rosell DR, Wood GE, Spedding M, Muñoz C, Rothstein $\mathrm{J}$ et al (2004). Chronic restraint stress up-regulates GLT-1 mRNA and protein expression in the rat hippocampus: reversal by tianeptine. Proc Natl Acad Sci USA 101: 2179-2184.

Roberts AC, Robbins TW, Everitt BJ, Muir JL (1992). A specific form of cognitive rigidity following excitotoxic lesions of the basal forebrain in marmosets. Neuroscience 47: 251-264.

Rogers MA, Kasai K, Koji M, Fukuda R, Iwanami A, Nakagome K et al (2004). Executive and prefrontal dysfunction in unipolar depression: a review of neuropsychological and imaging evidence. Neurosci Res 50: 1-11.

Ruis MAW, te Brake JHA, Buwalda B, De Boer SF, Meerlo P, Korte SM et al (1999). Housing familiar male wildtype rats together reduces the long-term adverse behavioural and physiological effects of social defeat. Psychoneuroendocrinology 24: 285-300.

Rygula R, Abumaria N, Flugge G, Fuchs E, Ruther E, HavemannReinecke U (2005). Anhedonia and motivational deficits in rats: impact of chronic social stress. Behav Brain Res 162: 127-134.

Sheline YI (2003). Neuroimaging studies of mood disorder effects on the brain. Biol Psychiatry 54: 338-352.

Silva RCB, Brandão ML (2000). Acute and chronic effects of gepirone and fluoxetine in rats tested in the elevated plus-maze: an ethological analysis. Pharm Biochem Behav 65: 209-216.

Stuss DT, Levine B, Alexander MP, Hong J, Palumbo C, Hamer L et al (2000). Wisconsin Card Sorting Test performance in patients with focal frontal and posterior brain damage: effects of lesion location and test structure on separable cognitive processes. Neuropsychologia 38: 388-402.

Szabo ST, Blier P (2001). Effect of the selective noradrenergic reuptake inhibitor reboxetine on the firing activity of noradrenaline and serotonin neurons. Eur J Neurosci 13: 2077-2087.

Szabo ST, de Montigny C, Blier P (2000). Progressive attenuation of the firing activity of locus coeruleus noradrenergic neurons by sustained administration of selective serotonin reuptake inhibitors. Int J Neuropsychopharmacol 3: 1-11.

Tollefson GD (1996). Cognitive function in schizophrenic patients. J Clin Psychiatry 57: 31-39.

Tunbridge EM, Bannerman DM, Sharp T, Harrison PJ (2004). Catechol-O-Methyltransferase inhibition improves set-shifting performance and elevates stimulated dopamine release in the rat prefrontal cortex. J Neurosci 24: 5331-5335.

Valentino RJ, Curtis AL, Parris DG, Wehby RG (1990). Antidepressant actions on brain noradrenergic neurons. J Pharmacol Exper Therap 253: 833-840.

Vyas A, Bernal S, Chattarji S (2003). Effects of chronic stress on dendritic arborization in the central and extended amygdala. Brain Res 965: 290-294.

Willner P (1997). Validity, reliability and utility of the chronic mild stress model of depression: a 10-year review and evaluation. Psychopharmacology 134: 319-329.

Willner P, Lappas S, Cheeta S, Muscat R (1994). Reversal of stressinduced anhedonia by the dopamine receptor agonist, pramipexole. Psychopharmacology (Berl) 115: 454-462.

Willner P, Mitchell PJ (2002). The validity of animal models of predisposition to depression. Behav Pharmacol 13: 169-188.

Willner P, Moreau J-L, Nielsen CK, Papp M, Sluzewska A (1996). Decreased hedonic responsiveness following chronic mild stress is not secondary to loss of body weight. Physiol Behav 60: $129-134$.

Willner P, Towell A, Sampson D, Sophokleous S, Muscat R (1987). Reduction of sucrose preference by chronic unpredictable mild stress, and its restoration by a tricyclic antidepressant. Psychopharmacology 93: 358-364. 